\title{
3D Profilometer Combined with Hyperspectral Camera for Simplified Rheumatoid Arthritis Diagnostics
}

\author{
Urban PAVLOVČIČ*1, Jošt STERGAR², Luka ROGELJ², \\ Jure KOŠIR ${ }^{1}$, Matija JEZERŠEK ${ }^{1}$, Matija MILANIČ2,3 \\ ${ }^{1}$ Faculty of Mechanical Engineering, University of Ljubljana, Ljubljana, Slovenia \\ ${ }^{2}$ Faculty of Mathematics and Physics, University of Ljubljana, Ljubljana, Slovenia \\ ${ }^{3}$ Jozef Stefan Institute, Ljubljana, Slovenia
}

DOI: $10.15221 / 18.031 \quad$ http://dx.doi.org/10.15221/18.031

\begin{abstract}
Rheumatoid arthritis is a disease, which significantly impairs patient's quality of life and ability to work. It has been proven that early diagnosis is of paramount importance, since early treatment has a higher likelihood for improving the course of the disease. Thus, the onset of the disease should be detected as early as possible. Changes in tissue oxygenation, blood concentration, light scattering and joint shape indicate joint inflammation, which could be detected and quantified using optical techniques. That is why a proposed system combines the hyperspectral imaging system and 3D profilometer. It enables measuring a spectrum of the reflected light from small joints with about $1 \mathrm{~nm}$ resolution, and a shape of the surface with a precision of about $0.02 \mathrm{~mm} \times 0.13 \mathrm{~mm} \times 0.02 \mathrm{~mm}$. In this study a RA diagnostics prototype comprising of a hyperspectral imaging system and a 3D scanning system is used to aid the rheumatoid arthritis diagnostics.
\end{abstract}

Keywords: 3d body scanning, rheumatoid arthritis, surface digitalization, biometrics, triangulation

\section{Introduction}

Rheumatoid arthritis (RA) is a destructive inflammatory polyarthritis with a prevalence of $1-2 \%$, that increases with age. RA leads to joint destruction and deformation, which may ultimately lead to loss of function and severely affects the patient's quality of life. The course of the disease is strongly dependent on the time lag between the onset and the beginning of the treatment.

Several studies have shown that there is a window of opportunity in the first year and especially in the first 3 months, in which the treatment may improve the course of the disease the most [1]. That is why, the early diagnosis and therapeutic intervention are of paramount importance.

Nowadays techniques like $\mathrm{x}$-ray, ultrasound, scintigraphy and magnetic resonance are used for arthritis diagnostics [2]. Unfortunately, these techniques have numerous drawbacks such as the need for ionizing radiation, low sensitivity, high variability between operators, and high costs.

In has been demonstrated that a hyperspectral imaging (HSI) may serve as an imaging modality for the diagnostics and follow-up monitoring of arthritis in small human joints. Typical symptom of RA is joint inflammation, which results in changes of tissue oxygenation, blood concentration, light scattering and joint shape and volume [2, 3, 4]. Moreover, a simple RGB camera system combined with illumination of the skin with $473 \mathrm{~nm}, 532 \mathrm{~nm}$ and $659 \mathrm{~nm}$ laser LEDs can provide distribution maps of skin melanin, oxy- and deoxyhemoglobin [5]. Similarly, using $460 \mathrm{~nm}, 535 \mathrm{~nm}$ and $663 \mathrm{~nm}$ illumination may provide hemoglobin and melanin concentration [6].

The main purpose of the proposed system is to explore possibility of applying a multispectral reflectance imaging to RA diagnostics, since it may detect various symptoms that accompany the arthritis diseases. The 3D profilometer is included to enable the correction of the HSI reflectance at locations with different heights and curvatures. In addition, it also provides volumetric information of the inspected joints. The imaging system is primarily designed to aid diagnosis of RA in human hands, so the measuring ranges are in line with the expected dimension of human hands. In the following sections, both modules of the system are presented in more detail and the results of preliminary test are shown. 


\section{Methods}

\subsection{D and HSI measuring}

The imaging system is composed of two modules. The first one, a 3D profilometer (3DP), provides the 3D information about the geometry of the measured surface, and the second one, an HSI system, provides the information about the spectrum of light reflected from a sample. The HSI system is composed of an imaging spectrograph (ImSpector V10E, Specim, Finland), a monochromatic camera (Blackfly S, BFS-U3-51S5M-C, 5 MP, FLIR Integrated Imaging Solutions, Canada), a $17 \mathrm{~mm}$ lenses (Xenoplan, 1.4/47-0903, Schneider-Kreuznach, Germany), and a custom multi-LED reflectance light source. The light source includes two multispectral LED arrays, where each of the arrays is mounted on the opposite side of the camera and tilted to illuminate the center of the camera field of view. The different types of LEDs are placed in the array in a special pattern which provides a homogenous illumination of the inspected area for each of the LED type. The homogenous illumination is achieved by an appropriate rod lens in front of the array. The spectral range of the system is $400 \mathrm{~nm}-1000 \mathrm{~nm}$ with roughly $1 \mathrm{~nm}$ spectral resolution, and a lateral spatial dimension $20 \mathrm{~cm}$ with about $200 \mu \mathrm{m}$ resolution.

The 3DP is based on a triangulation technique. It consists of a monochromatic camera (Flea3, FL3-U313Y3M-C, 1.3 MP, FLIR Integrated Imaging Solutions, Canada), $16 \mathrm{~mm}$ lens and a laser projector (FLEXPOINT, $30 \mathrm{~mW}, 405 \mathrm{~nm}$ wavelength, $60^{\circ}$ fan angle). The triangulation angle between optical axes of camera and projector is $56^{\circ}$ and baseline is $495 \mathrm{~mm}$.

The laser line illuminates a sample surface about $15 \mathrm{~mm}$ in front of the HSI system measuring region. This distance ensures, that the laser projector's light doesn't affect the reflected spectrum acquired by the HSI system. Both systems acquire only one spatial line at the time, thus to obtain the geometry and spectral response of the entire surface, the systems are mounted onto a linear translation stage providing horizontal scanning. A vertical translation stage is used to vary the height of both systems with respect to the base plate, so that measured object is always in focus of both systems.

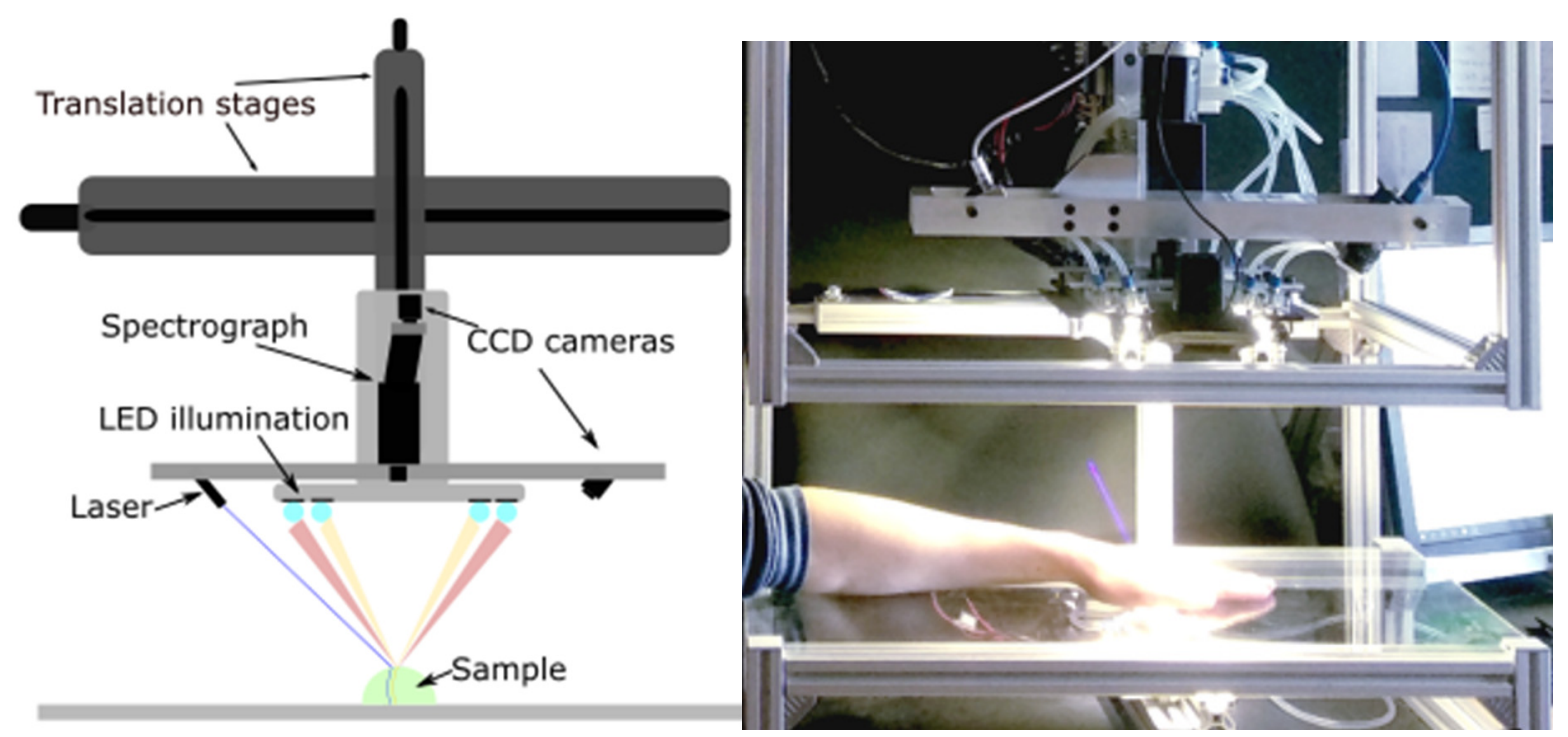

Figure 1. Schematics of the developed system (left) and real system in use (right).

The software component to drive translation stages, set, trigger and capture images from cameras, reconstruction, aligning and postprocessing were written in MATLAB, with additions of $\mathrm{C}_{++}$and OpenCV for performance critical tasks.

The 3DP was calibrated by measuring the reference plate of known geometry and optimizing the reconstruction parameters. After the calibration the precision of 3DP (one standard deviation of displacements between measured and ideal surface) was about $0.02 \mathrm{~mm} \times 0.13 \mathrm{~mm} \times 0.02 \mathrm{~mm}$ in X, $\mathrm{Y}$ and $\mathrm{Z}$ direction, respectively. Both measurements, the 3D geometry and HSI image, were aligned using a checkerboard. The final displacement between the spectral and 3D image was approx. $0.2 \mathrm{~mm}$. The imaging results are a hyperspectral cube (containing spectral information for each pixel of the image) and a 3D scan of the imaged hand. The 3D measurements enable determination of the joint volume, which typically increases due to the joint inflammation. Moreover, it allows for the joint thickness and curvature correction in the recorded HSI images. The uncorrected HSI images show brightness variation at locations with different elevations and curvatures, making the image analysis less accurate. 


\section{Results}

Several tests of the developed system were performed to prove the concept which would be implemented for the clinical diagnostics. Sample measurements of human fingers obtained by the multimodal system are shown in figure 2, showing ability of the system to determine joint volumes and correct the hyperspectral images. Here, an RGB image reconstructed from the HSI image (400$1000 \mathrm{~nm}$ range) is superimposed on the 3D geometry obtained by the 3DP (figure 2, left). The reflected spectrum is the combination of the tissues reflectance and the light source spectrum. Before an analysis of the $\mathrm{HSI}$ can be performed, such combined spectrum is corrected for the light source spectrum using a reference measurement on a sample (i.e., spectralon) with known reflectance.
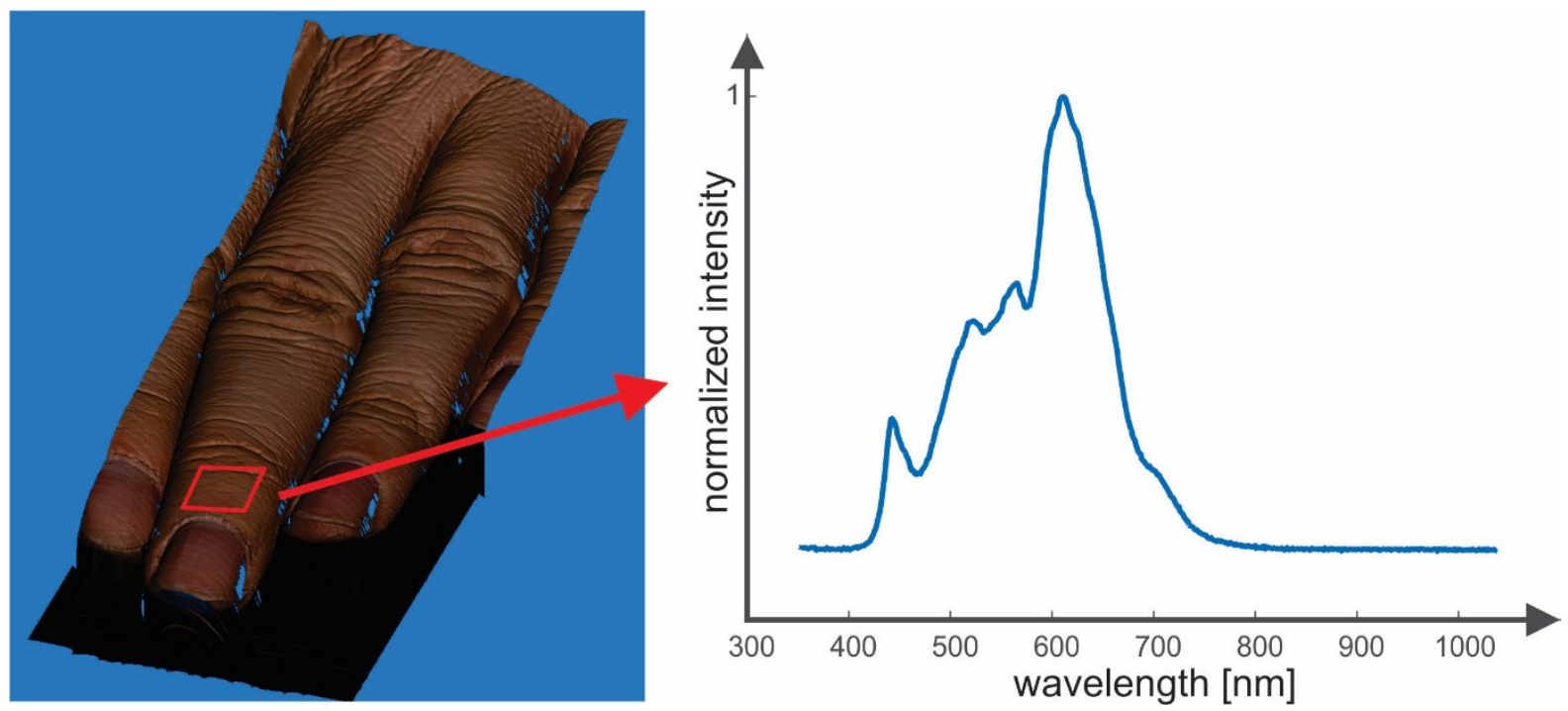

Figure 2. 3D shape of the measured surface with the superposed RGB image(left) and the spectrum of reflected light inside of the red rectangle (see the left figure).

Light source spectrum corrected reflectance spectra of a forearm during a blood flow occlusion test are shown in figure 3 . An occlusion test, also called a cuff-test, is commonly performed to induce hematologic physiological changes in studied tissues. A cuff is placed on the observed limb, typically on the upper arm, and pressure in the cuff is increased above arterial pressure. Consequentially blood flow in the affected limb stops and oxygen saturation of blood is gradually reduced. After the cuff is removed, an increased blood flow of oxygen saturated blood re-perfuses the limb, resulting in increased concentration of oxygen saturated blood.

Absorption spectra of the most important absorbers in human joints are presented in figure 3 (left). The occlusion test affects primarily the hemoglobin $(\mathrm{Hb})$ concentration. The saturated hemoglobin (oxy-Hb, red line) shows distinct peaks in the 530-590 nm region not present in the deoxygenated hemoglobin (deoxy-Hb, blue). The variation of hemoglobin species concentration during the occlusion test reflects well in the recorded spectra. Specifically, during the blood flow occlusion test the oxy-Hb peak at $560 \mathrm{~nm}$ disappears indicating increasing concentration of deoxy- $\mathrm{Hb}$ in the blood. Three minutes after the cuff is released the reflectance spectra are reduced and a pronounced $560 \mathrm{~nm}$ peak reappears. This indicates the increased amount of well oxygenated blood in the inspected tissue. Four minutes after the occlusion, the spectrum is slowly returning to the initial state, showing that the hematologic state returned to the baseline. It usually takes couple of minutes for spectrum to fully return in the initial state. 

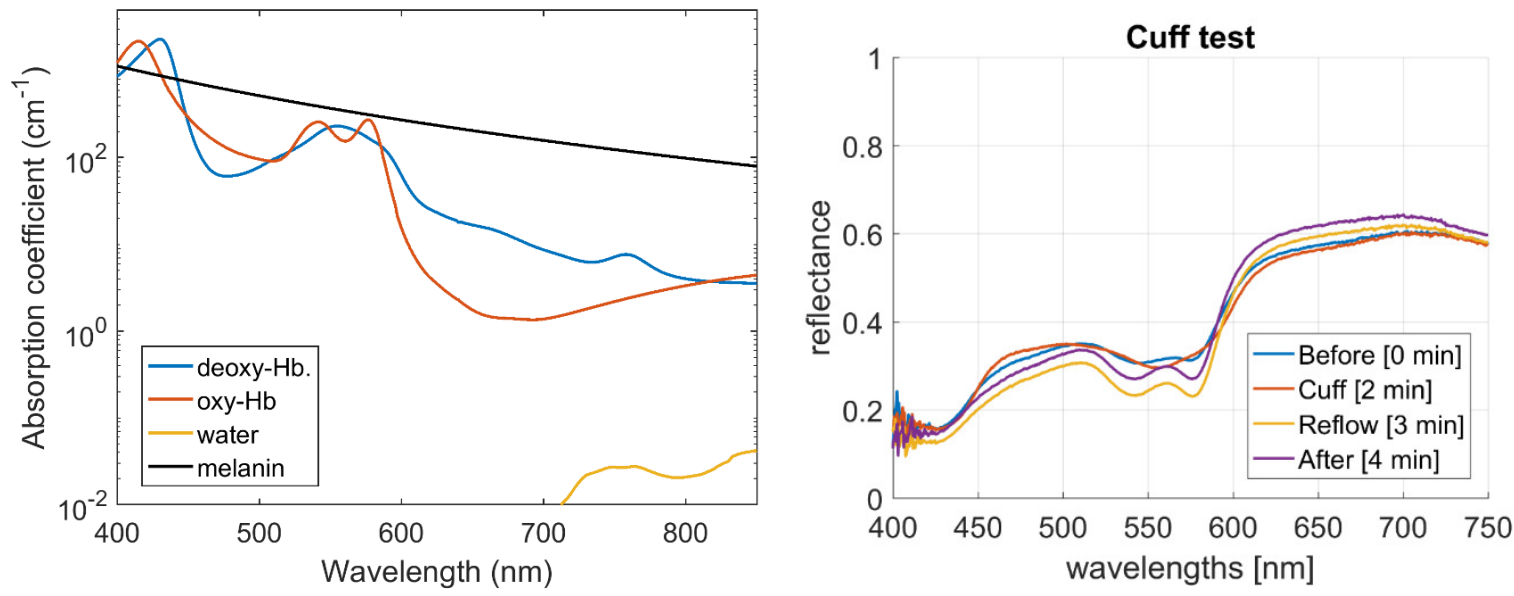

Figure 3. Absorption spectra of the most important tissue chromophores [7] (left) and reflectance spectra before, during and after the blood flow occlusion test.

Figure 4 shows results of another hematological test, where the inspected skin was irradiated by infrared light heating the tissue. The irradiation results in skin erythema, caused by hyperemia (increased blood flow) in the superficial skin capillaries.
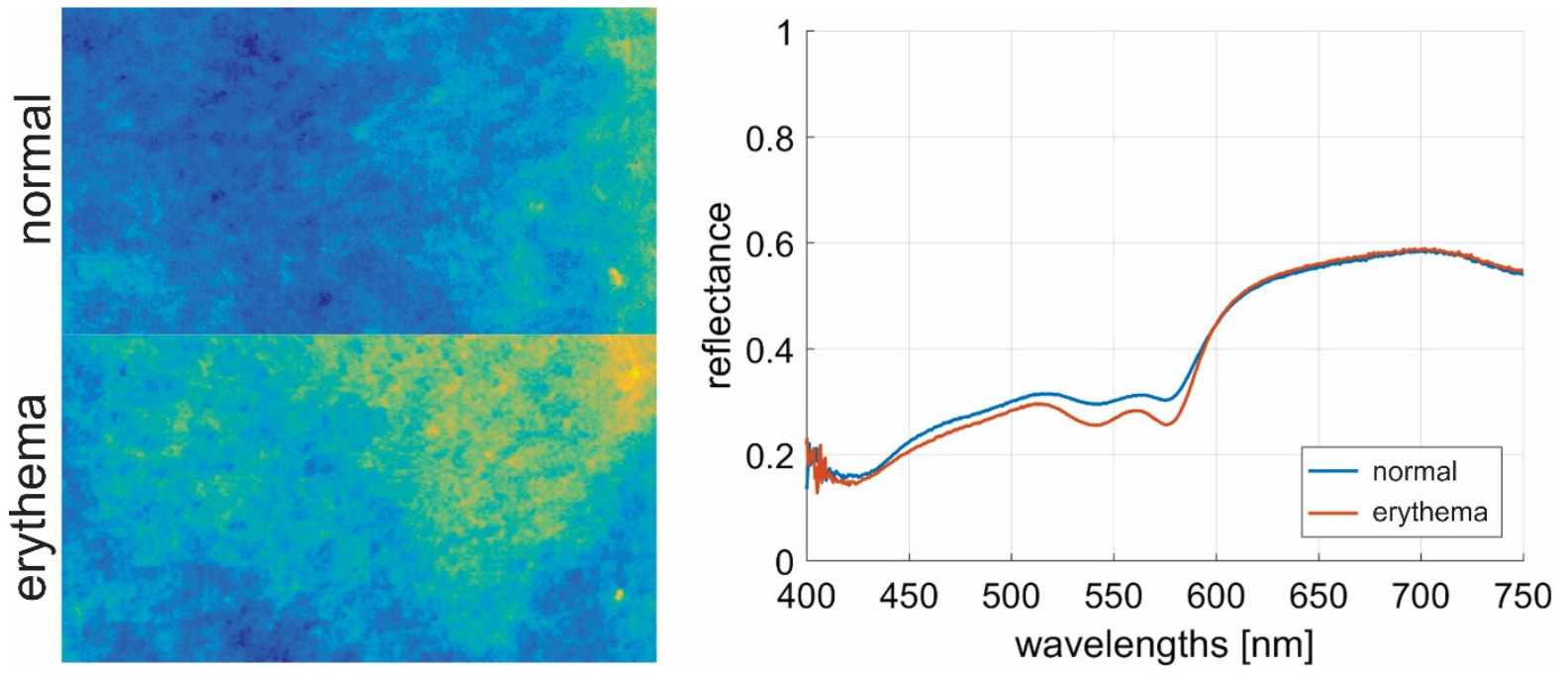

Figure 4. Images of normal skin (top left) and erythema (bottom left) and reflectance specters (right).

Similar to the occlusion test, the increased blood flow affects the measured reflectance spectrum by decreasing the reflectance in the 530-590 nm spectral region (figure 4, right) due to the increased concentration of the oxyhemoglobin. The recorded HSI was analyzed using the erythema index (EI) [8], an empirical index combining a few monochromatic images at various wavelengths sensitive to changes in blood concentration. El images (figure 4, left) shows regions where the IR irradiation induced increased blood perfusion (regions with increased yellow color).

\section{Conclusion}

An imaging system for RA diagnostics was developed. It is composed of a 3D profilometer, with precision of about $0.02 \mathrm{~mm} \times 0.13 \mathrm{~mm} \times 0.02 \mathrm{~mm}$, and a hyperspectral imaging system, with spectral resolution of about $1 \mathrm{~nm}$. Using the 3D geometry of the inspected surface obtained by the 3D profilometer, the hyperspectral image intensity variation due to the surface curvature can be performed. A hyperspectral image provides information about changes in blood flow and concentration of tissue chromophores or tissue oxygenation, which may indicate the onset of the disease. 


\section{References}

[1] J. A. B. van Nies, A. Krabben, J. W. Schoones, T. W. J. Huizinga, M. Kloppenburg, A. H. M.van der Helm-van Mil, "What is the evidence for the presence of a therapeutic window of opportunity in rheumatoid arthritis? A systematic literature review," Ann Rheum Dis, 73(5), 861-870, 2014.

[2] D. Chamberland, Y. Jiang, X. Wang, "Optical imaging: new tools for arthritis," Integr Biol (Camb), 2(10), 496-509, 2010.

[3] M. Milanic, L. A. Paluchowski, L. L. Randeberg, "Hyperspectral imaging for detection of arthritis: feasibility and prospects," J Biomed Opt, 20(9), 2015.

[4] J. M. Mountz, A. Alavi, J. D. Mountz, "Emerging optical and nuclear medicine imaging methods in rheumatoid arthritis," Nat Rev Rheumatol, 8(12), 719-728, 2012.

[5] E. Alerstam, W. C. Y. Lo, T. D. Han, J. Rose, S. Andersson-Engels, L. Lilge, "Next-generation acceleration and code optimization for light transport in turbid media using GPUs", Biomed Opt Express, 1(2), 658-675, 2010.

[6] L. M. H. da Mota, B. A. Cruz, C. V. Brenol, I. A. Pereira, L. S. Rezende-Fronza, M. B. Bertolo, M. V. C. Freitas, N. A. da Silva, P. Louzada-Junior, R. D. N. Giorgio, R. A. C. Lima, R. A. Kairalla, A. D. Kawassaki, W. M. Bernardo, G. D. C. Pinheiro, "Guidelines for the diagnosis of rheumatoid arthritis", Rev Bras Reumatol, 53(2), 141-157, 2013.

[7] J. Stergar, M. Milanič, "Assessment of line illumination for hyperspectral imaging by a Monte Carlo simulation", in Diffuse Optical Spectroscopy and Imaging VI, H. Dehghani, ed., Vol. 10412 of SPIE Proceedings (Optical Society of America, 2017), paper 104120Z.

[8] I. Diebele, I. Kuzmina, A. Derjabo, J. Kapostinsh, A. Bekina, J. Spigulis, "Analysis of skin basalioma and melanoma by multispectral imaging” in Proc. SPIE, 8427, 842732. 